\title{
A THEOREM ON INDUCED REPRESENTATIONS
}

\author{
ROBERT J. BLATTNER ${ }^{1}$
}

In [1], we proved a criterion for the disjointness of two induced representations $U^{L}$ and $U^{M}$ of a Lie group $G$, where $L$ and $M$ are finite-dimensional unitary representations of compact subgroups $H$ and $K$, respectively, of $G$. The purpose of this paper is to improve this theorem by getting a stronger conclusion, while dropping the conditions that $G$ be a Lie group and $H$ be compact, and that $L$ and $M$ be finite-dimensional. Moreover, the restriction on $K$ is weakened to read: $G$ has arbitrarily small neighborhoods of the identity invariant under the adjoint action of $K$ on $G$. Finally, the proof given below is fairly elementary, while the proof in [1] is quite involved.

Notations and conventions: Let $\mathcal{u}$ be a topological vector space. $C(G, \mathcal{u})$ will denote the space of continuous functions from $G$ to $\mathcal{U}$, equipped with the topology of uniform convergence on compact subsets of $G$ while $C_{0}(G, \mathfrak{u})$ will denote the space of those $f \in C(G, \mathfrak{u})$ with compact support. If $\mathcal{U}$ is omitted it is understood that $\mathcal{U}=\mathbf{C}$. If $\mathfrak{U}_{1}$ is another topological vector space, $\mathcal{L}\left(\mathcal{u}, \mathfrak{u}_{1}\right)$ will denote the space of continuous linear maps from $\mathfrak{u}$ into $\mathfrak{u}_{1}$ equipped with the topology of bounded convergence. All integrations are with respect to right Haar measure. For any locally compact group $G, \delta_{G}$ will denote its modular function. If $f, g \in C_{0}(G), f \circ g$ will denote the convolution of $f$ and $g$, and $f^{*}$ is defined by $f^{*}(x)=\delta_{G}(x)^{-1} f\left(x^{-1}\right)^{-}$. If $L$ and $M$ are representations of $G, R(L, M)$ will denote the space of intertwining operators for $L$ and $M$ (see [3]), while $I(L, M)$ will denote the dimension of $R(L, M)$. For the definition of induced representation used below, see [1]. Finally, for any function $f$ on the group $G$ and any $x \in G, f_{x}$ and $f^{x}$ are defined by $f_{x}(y)=f\left(x^{-1} y\right)$ and $f^{x}(y)=f(y x)$.

TheOREM. Let $H$ and $K$ be closed subgroups of the locally compact group $G$. Let $L$ (resp. $M$ ) be a unitary representation of $H$ (resp. $K$ ) on the Hilbert space $\mathcal{V}$ (resp. W). Suppose that $G$ has arbitrarily small neighborhoods of the identity invariant under the adjoint action of $K$ on $G$. Let $\mathfrak{T}$ be the subspace of those $S \in C(G, \mathcal{L}(\mathcal{V}, \mathfrak{W}))$ such that

$$
S\left(\xi^{-1} x \eta\right)=\delta_{H}(\xi)^{-1 / 2} \delta_{G}(\xi)^{1 / 2} M_{\eta}^{-1} S(x) L_{\xi}
$$

for all $\xi \in H, \eta \in K$, and $x \in G$. Then

$$
I\left(U^{L}, U^{M}\right) \leqq \operatorname{dim} \mathfrak{T},
$$

provided $\operatorname{dim} \mathfrak{T}$ is finite.

Received by the editors, November 10, 1961 .

1 National Science Foundation Fellow. 
Proof. We recall from [1] the bilinear map $\epsilon_{H}$ defined on $C_{0}(G) \times v$ with values in the Hilbert space of $U^{L}$ :

$$
\epsilon_{H}(f, v)(x)=\int_{H} \delta_{H}(\xi)^{-1 / 2} \delta_{G}(\xi)^{1 / 2} f(\xi x) L_{\xi}^{-1} v d \xi
$$

for $f \in C_{0}(G), v \in \mathcal{V}$. $\epsilon_{K}$ is similarly defined from $C_{0}(G) \times W$ into the space of $U^{M}$. Given $f, g \in C_{0}(G), v \in \mathcal{V}$, and $w \in W$, we define the linear functional $\Phi(f, g, v, w)$ on $R\left(U^{L}, U^{M}\right)$ by setting $\Phi(f, g, v, w)(A)$ $=\left(A \epsilon_{H}(f, v), \epsilon_{K}(g, w)\right)$ and the linear functional $\Psi(f, g, v, w)$ on $\mathfrak{T}$ by setting

$$
\Psi(f, g, v, w)(S)=\int_{G}\left(f \circ g^{*}\right)(x)(S(x) v, w) d x
$$

$\Phi$ and $\Psi$ are linear in $f$ and $v$ are are conjugate-linear in $g$ and $w$.

Let $S$ (resp. $J$ ) be the linear space of functionals spanned by the $\Phi$ 's (resp. $\Psi^{\prime}$ ) $)$. It is obvious that $\mathfrak{I}$ separates $\mathfrak{T}$. That $\mathcal{S}$ separates $R\left(U^{L}, U^{M}\right)$ follows from [1, Lemma 2]. We shall show that there is a linear map of $\Im$ onto $\$ which sends each $\Psi(f, g, v, w)$ onto $\Phi(f, g, v, w)$, and this will accomplish the proof.

According to $[1$, Lemma 2$]$, there is a constant $C_{Q}$ for each compact set $Q$ in $G$ such that

$$
|\Phi(f, g, v, w)(A)| \leqq C_{Q}\|f\|_{\infty}\|g\|_{\infty}\|v\|\|w\|\|A\|
$$

whenever $f$ and $g$ have their supports in $Q$. From this it follows easily that for each $h \in C_{0}(G), A \in R\left(U^{L}, U^{M}\right)$, and $x \in G$ there is an operator $T_{h}(A)(x) \in \mathfrak{L}(\mathcal{V}, w)$ such that $\left(T_{h}(A)(x) v, w\right)=$ $\delta_{G}(x)^{-1} \Phi\left(h_{x}, h, v, w\right)(A)$ for all $v \in v$ and $w \in W$ and that $T_{h} \in \mathcal{L}\left(R\left(U^{L}, U^{M}\right), C(G, \mathfrak{L}(\mathcal{\mho}, \mathscr{W}))\right)$.

Now the standard proof of the complete regularity of $G$ (cf. [4, pp. 28-30]) may be modified to show that for any neighborhood $N$ of $e$ in $G$, there is a nonzero positive continuous function $h$ whose support is in $N$ such that $h(\eta x)=h(x \eta)$ for all $x \in G$ and $\eta \in K$ : one need merely require all neighborhoods of $e$ mentioned therein to be invariant under the adjoint action of $K$ on $G$. Let $h$ be such a $K$ invariant function. For every $\eta \in K, \delta_{G}(\eta) \int_{G} h(x) d x=\int_{G} h\left(\eta^{-1} x\right) d x$ $=\int_{G} h\left(x \eta^{-1}\right) d x=\int_{G} h(x) d x$. Since $\int_{G} h(x) d x>0$, it follows that $\boldsymbol{\delta}_{G} \mid K$ $=1$. Similarly $\delta_{K}=1$.

If $h$ is a $K$-invariant function in $C_{0}(G)$, then $T_{h}(A) \in \mathfrak{T}$ for all $A \in R\left(U^{L}, U^{M}\right)$. In fact, $\epsilon_{H}\left(f_{\xi^{-1}}, v\right)=\delta_{H}(\xi)^{-1 / 2} \delta_{G}(\xi)^{-1 / 2} \epsilon_{H}\left(f, L_{\xi} v\right)$ and $\epsilon_{H}\left(f^{x}, v\right)=U_{x}^{L} \epsilon_{H}(f, v)$ for all $f \in C_{0}(G), v \in v, \xi \in H$, and $x \in G$ (cf. the proof of Theorem 3 in [1]). Similar statements hold for $\epsilon_{K}$. Also 
$h_{\eta}=h^{\eta^{-1}}$ for $\eta \in K$. Therefore

$$
\begin{aligned}
\left(T_{h}(A)\left(\xi^{-1} x \eta\right) v, w\right) & =\delta_{G}\left(\xi^{-1} x \eta\right)^{-1} \Phi\left(h_{\xi}^{-1} x \eta, h, v, w\right)(A) \\
& =\delta_{H}(\xi)^{-1 / 2} \delta_{G}(\xi)^{1 / 2} \delta_{G}(x)^{-1} \Phi\left(h_{x}^{\eta^{-1}}, h, L_{\xi} v, w\right)(A) \\
& =\delta_{H}(\xi)^{-1 / 2} \delta_{G}(\xi)^{1 / 2} \delta_{G}(x)^{-1} \Phi\left(h_{x}, h^{\eta}, L_{\xi} v, w\right)(A) \\
& =\delta_{H}(\xi)^{-1 / 2} \delta_{G}(\xi)^{1 / 2} \delta_{G}(x)^{-1} \Phi\left(h_{x}, h, L_{\xi} v, M_{\eta} w\right)(A) \\
& =\delta_{H}(\xi)^{-1 / 2} \delta_{G}(\xi)^{1 / 2}\left(M_{\eta}^{-1} T_{h}(A)(x) L_{\xi} v, w\right)
\end{aligned}
$$

for all $\xi \in H, \eta \in K, x \in G, v \in \mathcal{V}$, and $w \in W$, as claimed.

We next assert that $\Psi(f, g, v, w) \circ T_{h}=\Phi\left(f, h \circ h^{*} \circ g, v, w\right)$ for $f, g, h \in C_{0}(G), v \in \mathcal{V}, w \in W$, where $h$ is $K$-invariant. To see this, note that $f \circ g=\int g(x) f^{x^{-1}} d x=\int f\left(x^{-1}\right) g_{x^{-1}} d x$, where the integrals on the right are strong integrals in the uniform topology. Using the continuity mentioned above of $\Phi$ in its arguments, we deduce that

$$
\begin{aligned}
\Psi(f, g, v, w)\left(T_{h}(A)\right) & =\int\left(f \circ g^{*}\right)\left(x^{-1}\right) \Phi\left(h_{x}{ }^{-1}, h, v, w\right)(A) d x \\
& =\Phi\left(\int\left(f \circ g^{*}\right)\left(x^{-1}\right) h_{x}{ }^{-1} d x, h, v, w\right)(A) \\
& =\Phi\left(f \circ g^{*} \circ h, h, v, w\right)(A) \\
& =\Phi\left(\int\left(g^{*} \circ h\right)(x) f^{x^{-1}} d x, h, v, w\right)(A) \\
& =\int\left(g^{*} \circ h\right)(x) \Phi\left(f^{-1}, h, v, w\right)(A) d x \\
& =\int\left(g^{*} \circ h\right)(x) \Phi\left(f, h^{x}, v, w\right)(A) d x \\
& =\Phi\left(f, \int\left[\left(g^{*} \circ h\right)(x)\right]-h^{x} d x, v, w\right)(A) \\
& =\Phi\left(f, h \circ\left(g^{*} \circ h\right)^{*}, v, w\right)(A),
\end{aligned}
$$

as claimed.

Finally, let $\lambda_{\text {fovw }}$ be a set of complex numbers indexed by $C_{0}(G)$ $\times C_{0}(G) \times V \times w$, all but a finite number of which vanish, such that $\sum \lambda_{f g v w} \Psi(f, g, v, w)=0$. Then $\sum \lambda_{f g v w} \Phi\left(f, h \circ h^{*} \circ g, v, w\right)=0$ for all $K$-invariant $h \in C_{0}(G)$. Letting $h \circ h^{*}$ approach the $\delta$-measure, we see that $\sum \lambda_{f g v w} \Phi(f, g, v, w)=0$. Therefore the linear map of $\Im$ onto $S$ mentioned at the beginning of this proof exists, and our proof is complete. 
For $x \in G$, we define a representation $M^{x}$ of $x K x^{-1}$ by setting $M_{\eta}^{x}=M_{x}^{-1}{ }_{\eta x}$ for all $\eta \in x K x^{-1}$. It is easy to see that

$$
I\left(\delta_{H}^{-1 / 2} \delta_{G}^{1 / 2} L\left|H \cap x K x^{-1}, M^{x}\right| H \cap x K x^{-1}\right)
$$

depends only on $L, M$, and the $H: K$ double coset $D$ to which $x$ belongs (cf. [3]). We shall denote this number by $I(L, M, D)$. In what follows, $D$ will denote the set of $H: K$ double cosets. We retain the hypotheses of the Theorem.

Corollary 1. Let $D_{0}$ be the set of open $D \in D$. Suppose that $\cup\left\{D: D \in D_{0}\right\}$ is dense in $G$. Then $I\left(U^{L}, U^{M}\right) \leqq \sum\left\{I(L, M, D): D \in D_{0}\right\}$, provided this sum is finite.

(A simple category argument shows that the denseness hypothesis is satisfied if $H$ and $K$ are denumerable at infinity and $D$ is denumerable. Cf. [2, Lemma $3 ; 1$ ].)

Proof. To each $D \in D$, assign an element $x_{D} \in D$. Let

$$
R=\prod\left\{R\left(\delta_{H}^{-1 / 2} \delta_{G}^{1 / 2} L\left|H \cap x_{D} K x_{D}^{-1}, M^{x_{D}}\right| H \cap x_{D} K x_{D}^{-1}\right): D \in D_{0}\right\} .
$$

For each $S \in \mathscr{T}$, let $\Lambda(S)$ be the function from $\mathscr{D}_{0}$ to $\mathscr{L}(\mathcal{V}, \mathcal{W})$ defined by $\Lambda(S)(D)=S\left(x_{D}\right), D \in D_{0} . \Lambda$ is linear. It is also $1-1$, since $\Lambda(S)=0$ implies that $S \mid D=0$ if $D \in D_{0}$ so that $S$ vanishes on a dense subset of $G$. Finally, $\operatorname{Im}(\Lambda) \subseteq R$. In fact, let $\xi \in H \cap x_{D} K x_{D}^{-1}$. Then $x_{D}^{-1} \xi x_{D} \in K$ and $S\left(x_{D}\right)=S\left(\xi^{-1} x_{D}\left(x_{D}^{-1} \xi x_{D}\right)\right)=\delta_{H}^{-1 / 2}(\xi) \delta_{G}(\xi)^{1 / 2} M_{x_{D}-1 \xi x_{D}} S\left(x_{D}\right) L_{\xi}$, whence our assertion. The corollary is an immediate consequence of the Theorem and the properties of $\Lambda$.

Corollary 2. Let $D_{n}$ be the set of $D \in D$ for which $I(L, M, D)=0$. Suppose that $\cup\left\{D: D \in D_{n}\right\}$ is dense in $G$. Then $I\left(U^{L}, U^{M}\right)=0$.

Proof. Let $S \in \mathbb{T}$. The methods used in proving Corollary 1 show that $S\left(x_{D}\right)=0$ for $D \in D_{n}$, whence $S$ vanishes on a dense subset of $G$ and we have our result.

\section{REFERENCES}

1. Robert J. Blattner, On induced representations, Amer. J. Math. 83 (1961), 79-98.

2. F. Bruhat, Sur les représentations induites des groupes de Lie, Bull. Soc. Math. France 84 (1956), 97-205.

3. G. W. Mackey, Induced representations of locally compact groups. I, Ann. of Math. 55 (1952), 101-139.

4. D. Montgomery and L. Zippin, Topological transformation groups, Interscience, New York, 1955.

Massachusetts Institute of TechNology aNd University of California, Los Angeles 